\title{
A model of an aircraft towing a cable-body system
}

\author{
$\begin{array}{ll}\text { C. K. H. Chin } & \text { R. L. May }\end{array}$
}

(Received 2 November 2005; revised 31 January 2007)

\begin{abstract}
We integrate together a model of an aircraft with a model of a body being towed by a thin elastic cable. The model of the towed body allows for either payout or retrieval of the cable. Both the aircraft and towed body are modelled as rigid bodies having six degrees of freedom. All aerodynamic forces and moments acting on the bodies are approximated linearly and so are expressed in terms of stability coefficients. A variable time step was employed for the aircraft model and a fixed time step was used for the towed body model; the integrated model retains this feature. Results of simulated flights are presented for both a Cessna and a Boeing 747 towing a Hayes TRX-12 target.
\end{abstract}

*Department of Maritime Engineering, Australian Maritime College, Launceston, Tasmania 7250, Australia. mailto: c. chin@amc.edu.au

${ }^{\dagger}$ School of Mathematical and Geospatial Sciences, RMIT University, Melbourne, Victoria 3000, Australia. mailto:rob@rmit.edu.au

See http://anziamj.austms.org.au/V47EMAC2005/Chin for this article, (C) Austral. Mathematical Soc. 2007. Published April 9, 2007. ISSN 1446-8735 


\section{Contents}

1 Introduction

C616

2 Coupling of models

C617

3 Equilibrium position

C619

4 Data

C623

5 Results

C624

6 Conclusions

C629

References

C629

\section{Introduction}

Since the early days of powered flight, aircraft have been towing bodies such as instruments, antennas, banners, gliders, and targets. In the last few decades, the practice of towing decoys has grown rapidly.

Chin, May \& Connell [4] modelled a body towed by a long thin elastic cable. The cable is assumed to be homogeneous, have no bending stiffness and have a uniform cross section in its unstretched state. The motion of the cable is described by a system of six partial differential equations, and a six degrees of freedom model of the module is employed. The usual linear approximation of the aerodynamic forces and moments acting on the module is used [6]. An implicit finite difference scheme is used to integrate the system of differential equations comprising of six equations for the cable and nine for the module. It employs a constant time step, and uses values at three time levels. Broydon's method [2, e.g.] is used to solve the resulting system of 
non-linear equations at each time step. The model allows for any prescribed payout or retrieval of the cable.

We also developed an aircraft model [3]. Again, a six degrees of freedom model is used with a linearization of the aerodynamic forces and moments. The system of nine ordinary differential equations is integrated using the adaptive Runge-Kutta-Fehlberg method [2]. A simple control system is incorporated to determine appropriate aileron, elevator, rudder and throttle adjustments to achieve a required response such as "bank left to $30^{\circ}$ at a rate of $5^{\circ}$ per second while maintaining the present altitude and speed". Such commands can be issued at any time and are included in an input file.

This article describes the coupling of the two models to produce an integrated model that simulates the flight of an aircraft towing a cable-module system for any sequence of commands and any combination of payout and retrieval of the cable. The results of simulations using this model are useful in the design of towing systems.

In all simulations the aircraft is assumed to be initially flying on a straight path at constant speed and altitude with the cable and module in their equilibrium position. The procedure to trim the aircraft to achieve this is discussed. Next some data for the cables and the module is presented, and finally some examples of the complete model are given.

\section{Coupling of models}

The effect of the attached cable had to be included in the aircraft model. The tension in the cable at the aircraft end is denoted by $T_{a}$ and its orientation is specified in terms of the two Euler angles $\phi_{a}$ and $\theta_{a}$ (the third Euler angle has the fixed value of $\pi / 2$ [4]). Contributions to the three components of force and three moments arising from the cable were added; of course these are dependent on the position of attachment of the cable. 
The aircraft model needs small time steps since control inputs may be made at any time and the state variables may change rapidly. However, the cable and module respond more slowly and thus bigger time steps are used. It is too expensive to use a small time step for the complete model, so it was decided to integrate the aircraft equations using the Runge-Kutta-Fehlberg method over intervals of length $\Delta t$, the fixed time step used for the cable and module equations.

Consider the interval $\left[t_{n}, t_{n+1}\right]$ where $t_{n}=n \Delta t$. When the aircraft model equations are integrated over this interval, values of the cable tension and Euler angles will be required at times within this interval. Approximate values of the tension $T_{a}$ and angles $\phi_{a}$ and $\theta_{a}$ are known at times $t_{n}, t_{n-1}, \ldots$, and are denoted by $T_{a}^{n}, T_{a}^{n-1}, \ldots$ It is easily shown that the quadratic that passes through the last three known values is

$$
\begin{aligned}
T_{a}(t)= & T_{a}^{n}+\left(t-t_{n-1}\right)\left(\frac{T_{a}^{n}-T_{a}^{n-1}}{\Delta t}\right) \\
& +\left(t-t_{n-1}\right)\left(t-t_{n}\right)\left(\frac{T_{a}^{n}-2 T_{a}^{n-1}+T_{a}^{n-2}}{2 \Delta t^{2}}\right) .
\end{aligned}
$$

This expression is evaluated to give an estimate of the tension at any required time $t$ in the interval $\left[t_{n}, t_{n+1}\right]$, and analogous expressions are used to give estimates of $\phi_{a}$ and $\theta_{a}$.

Once the aircraft equations have been integrated over the interval $\left[t_{n}, t_{n+1}\right]$, estimates of the velocity components at the aircraft end at time $t_{n+1}$ that are needed to integrate the cable equations are known. Thus the cable and module equations may be integrated over the interval to complete the time step.

A 'corrector' step (or steps) could be used. At the end of the time step, values of the tension and angles given by Equation (1) (and its counterparts for $\phi_{n}$ and $\theta_{n}$ ) could be compared with the values calculated from the cable equations, and if they were too different the step could be repeated, but this 
time including the data from the cable model in the estimate (a cubic could be fitted through the four data points). However, experimentation showed that the use of a corrector step made no appreciable change to the results.

\section{Equilibrium position}

The model is started (at $t=0$ ) with the aircraft flying in a straight and level flight at a specified speed and altitude with the cable and module in their equilibrium positions. If no control inputs or cable payout or retrieval are made, the configuration of the aircraft-cable-module system should stay the same; the only change will be in its position.

In order to find the initial values of all of the variables, the equilibrium position of the module must be determined first. This is done by setting the components of force acting on the module and the moments about three axes equal to zero. Of course the effect of the cable load is included in the force components and moments. Because the cable attachment point is assumed to lie in a vertical plane which contains the centreline of the module, this leads to three non-linear equations that are solved for three unknowns, the pitch angle of the module and the tension and angle of the cable. The cable tension is rather sensitive to the longitudinal position of the attachment point (see Figure 1). Moving the attachment point forward of the centre of mass causes the module to pitch up, giving some lift and thus reducing the tension in the cable. Conversely, moving it behind the centre of mass causes the module to pitch down, resulting in a downforce and thus increasing the tension in the cable. These results show that a small change in pitch angle can cause a large change in the tension. Obviously, the same applies for any yawing motion, and it is clear that in flight small changes in attitude will be reflected by larger changes in the tension of the cable.

Once the equilibrium position of the module has been found, the equilibrium position of the cable is calculated. Setting all time derivatives in the 


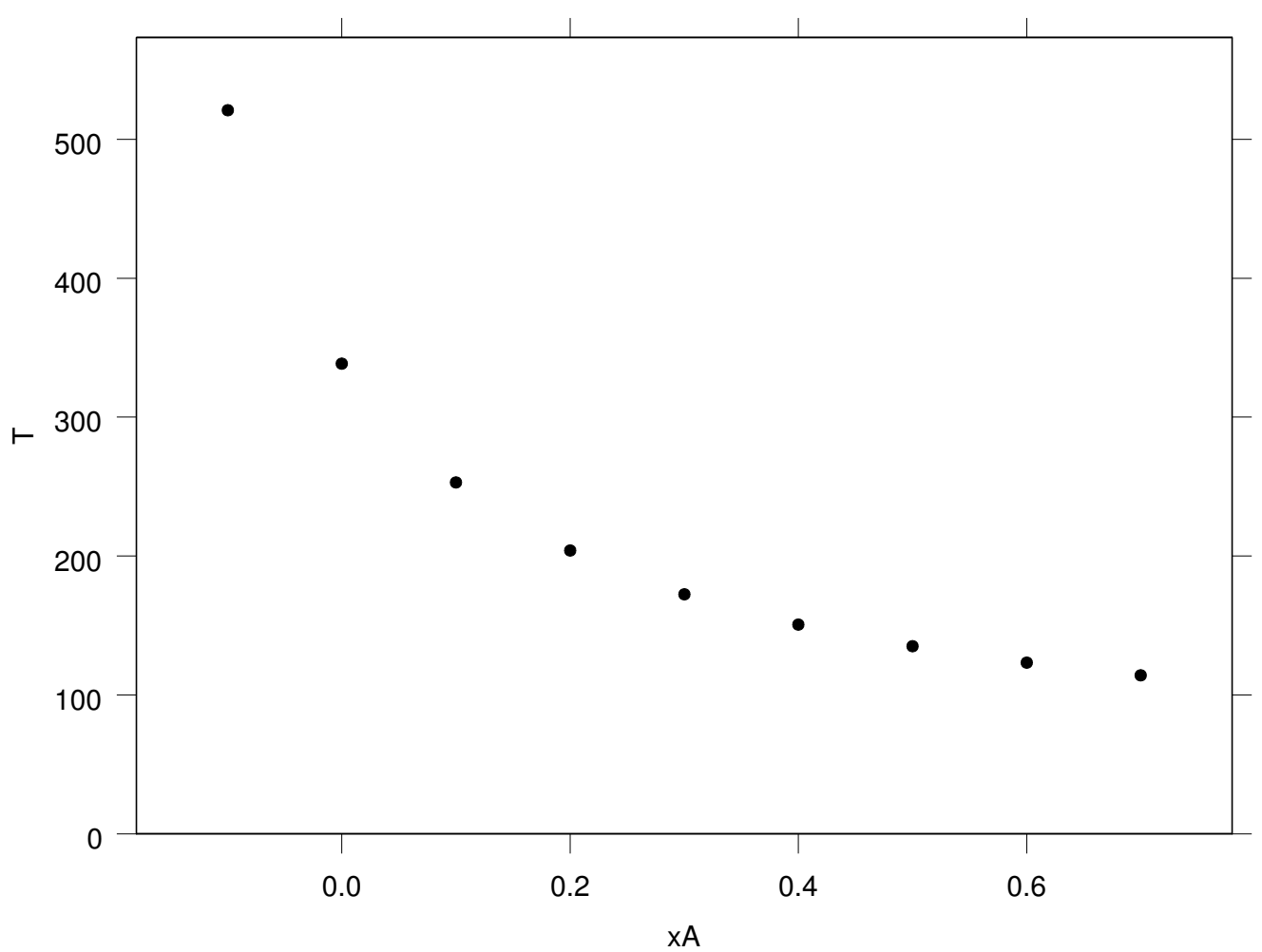

Figure 1: Tension of the cable at the module as the position of the attachment point is varied. The variable on the horizontal axis is the distance of the attachment point forward from the centre of mass. 
cable equations to zero and using the fact that the cable will lie in a vertical plane leaves a system of four ordinary differential equations which are solved using the Runge-Kutta-Fehlberg method to give the angles, tension and velocity components at the grid points on the cable.

The force exerted by the cable on the aircraft is now known, so the aircraft can be "trimmed" to fly at the specified (constant) speed and altitude. Again the force components and moments are set to zero, giving a system of six non-linear equations. These equations involve seven unknowns, the aileron, elevator and rudder deflections, the throttle position, and the pitch, roll and yaw angles, so there are infinitely many solutions. However, it was decided to trim the aircraft so that it has no yaw, so the solution of the equations will be unique.

Body axes are used with, at level straight flight with no cable loads, the $x$ axis pointing forwards, the $y$ axis horizontal and the $z$ axis pointing vertically downwards. The force components in these directions are denoted by $X, Y$ and $Z$ respectively, and the moments about the axes are denoted by $L, M$ and $N$. An iterative process is used, starting with all variables set to zero. First the equation $X=0$ is solved for the throttle position, then $Y=0$ for the roll angle, $Z=0$ for the pitch angle, $L=0$ for the aileron deflection, $M=0$ for the elevator deflection, and finally $N=0$ solved for the rudder deflection. This process is repeated until

$$
\left[\left(\frac{X}{\text { max thrust }}\right)^{2}+Y^{2}+\left(\frac{Z}{\text { Weight }}\right)^{2}+L^{2}+M^{2}+N^{2}\right]^{1 / 2} \leq \epsilon,
$$

where $\epsilon$ is a prescribed tolerance. The second and third equations can not be solved analytically for the variable in question, so the bisection method was used with a tolerance of $10^{-8}$.

This trimming procedure was tested for a Cessna and a Boeing 747; all the data for these aircraft was obtained from Roskam [6]. A load was attached in five different positions on the aircraft. Three were on the underside of the 


\begin{tabular}{|c|c|c|}
\hline$\varepsilon$ & No. of iterations & Deviation $(\mathrm{m})$ \\
\hline 1 & 4 & 212.4573 \\
$10^{-1}$ & 5 & 14.3645 \\
$10^{-2}$ & 6 & 0.9319 \\
$10^{-3}$ & 7 & 0.0604 \\
$10^{-4}$ & 8 & 0.0039 \\
$10^{-5}$ & 9 & 0.0003 \\
\hline
\end{tabular}

TABLE 1: Dependence of the deviation from straight and level flight on the tolerance $\varepsilon$.

fuselage, one near a point beneath the centre of mass, another forward of this point and one near the tail. The other two points were on the underside of the right wing, one about halfway along the wing and the other near the wing tip. For the Cessna, a load of $1000 \mathrm{~N}$ was applied at an angle of $10^{\circ}$ down from the horizontal, being typical of a load from a towed-cable system. When the load was applied to each of the points in turn there was only a very small change in the pitch angle and elevator angle, but in all cases, the throttle was increased by about $13 \%$ to maintain the correct speed. For the points on the fuselage, the aileron, rudder and roll angles were all zero, but attaching the load to the point near the end of the wing resulted in a rudder angle of about $11^{\circ}$, a roll angle of approximately $5.5^{\circ}$ and an elevator angle of around $1.2^{\circ}$. Not unexpectedly, the angles for the attachment point halfway along the wing were about half as large.

The algorithm was run for the Cessna with the load attached to the wing tip for different values of the tolerance $\varepsilon$. Table 1 shows the deviation from straight and level flight, calculated after 400 seconds of flight with no further changes being made to the controls after trimming and a constant cable force. Clearly the iteration converges rapidly, and the deviation is insignificant for $\varepsilon \leq 10^{-3}$.

If the aircraft is properly trimmed and the integrated model is run with no control inputs, then the aircraft should continue to fly in a horizontal 
straight path at a constant velocity, and the module and the cable should move with the same constant velocity. However, the cable equations are solved initially using a method of order six with small spatial steps, but during the time stepping, they are approximated only to second order with larger cable segments, so there will be a slight change in the shape of the cable as it adjusts to the equilibrium position dictated by the second-order spatial discretization of the cable equations. Once this "new" equilibrium position is reached, the module, cable and aircraft will move as a "unit" in a horizontal flight.

\section{Data}

The cables used in aerial towing are roughly circular in cross-section with diameters typically between 1 and $5 \mathrm{~mm}$, and lengths can be up to 10,000 $\mathrm{m}$ [7]. Cables are generally made of steel wire, and various constructions are used; cables can consist of a single wire (a monofilament cable) or be made up of a number of thinner wires twisted together.

In the simulations shown later a monofilament steel cable of diameter $d=2 \mathrm{~mm}$ is used. The mass per unit length $\tilde{m}$ and the parameter $e=1 / E A$, where $E$ is Young's modulus and $A$ is the area of cross section, are readily calculated to be $0.02466 \mathrm{~kg} \mathrm{~m}^{-1}$ and $1.592 \times 10^{-6}$ respectively.

The drag coefficients for the cable have to be specified. There have been numerous experiments to determine the effect of the Reynolds number on the drag coefficients for flow past a cylinder, and it would seem that for typical towing speeds and cable diameters, the normal coefficient $c_{d} \approx 1.2$ and the tangent drag coefficient $c_{t}$ lies in the range 0.01 and 0.03 [7]. Cochran et al. [5] give empirical formulae for the drag coefficients in terms of the Mach number, but there is no indication of the source of these formulae. At a tow speed of $150 \mathrm{~ms}^{-1}$, these formulae give the values $c_{d} \approx 1.2$ and $c_{t} \approx 0.02$ which are in agreement with the figures quoted above. Consequently, the 
values $c_{d}=1.2$ and $c_{t}=0.02$ have been used in the simulations in this article.

Wingham [7] states that typically towed modules have a mass in the range of 5 to 150 kilograms and are towed at speeds of between 50 and $210 \mathrm{~ms}^{-1}$. The only set of published data on a towed module found is in $[1,5]$ and is representative of the TRX-12 manoeuvrable towed target developed by Hayes International Targets. This data was used for the following simulations but movement of the control surfaces was not employed.

As stated above, all aircraft data is recorded by Roskam [6].

\section{$5 \quad$ Results}

The following simulations are carried out using a time step of 0.1 seconds, for a Cessna aircraft that is initially flying at an altitude of 9144 metres with a speed of $139 \mathrm{~ms}^{-1}$, or a Boeing aircraft initially flying at an altitude of 6096 metres with a speed of $205 \mathrm{~ms}^{-1}$. The cable is attached to the underside of the fuselage near the tail of each aircraft, and is attached to the module at the point $0.15 \mathrm{~m}$ forward of the centre of mass.

Considerable numerical experimentation has been performed to determine suitable parameters such as the time step and length of cable segments. For example, the time step was successively reduced until there was no appreciable difference between the calculated results and those using a larger step.

The first example is of for the Cessna. We start with a cable of length 720 metres that is modelled using $n=55$ nodes. The aircraft pitches down at $t=20$ seconds at an angle of $10^{\circ}$ at a rate of $2^{\circ}$ per second. Reeling in of the cable begins at $t=26$ seconds, and reaches its maximum retrieval rate of $15 \mathrm{~ms}^{-1}$ at $t=30$ seconds. The aircraft levels off at $t=30$ seconds and the thrust returned to its initial value. At time 60 seconds, the aircraft 


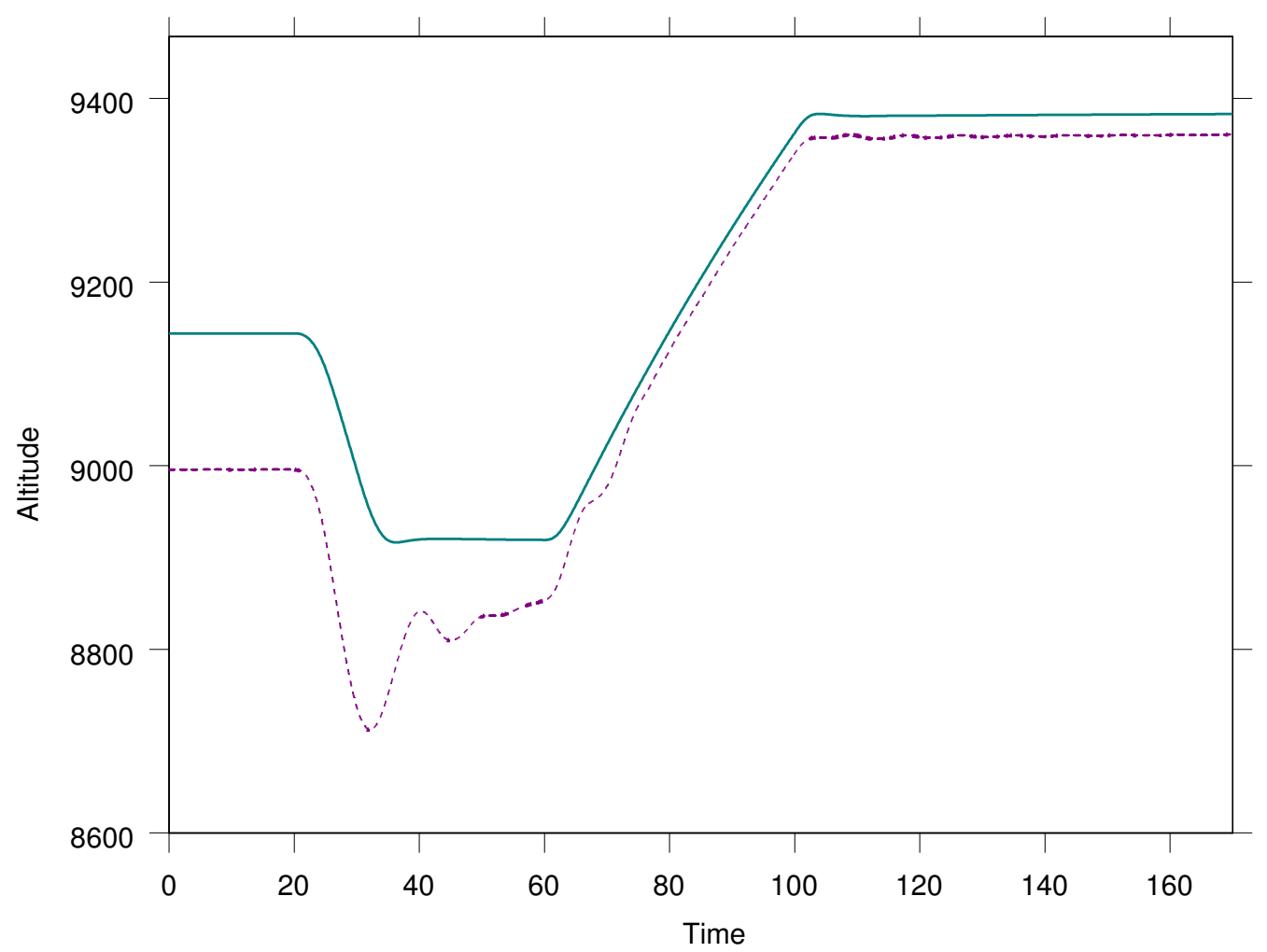

Figure 2: Variation of height over time of the Cessna aircraft (green solid line) and module (purple dashed line). 
pitches up at $5^{\circ}$ at $2^{\circ}$ per second with full power. Between $t=72$ and $t=76$ seconds, the retrieval velocity of the cable reduces to zero. At 100 seconds, the aircraft returns to level flight. This results in 30 metres of cable left with a stretched length of 30.011 metres with $n=33$ nodes. The velocity of the aircraft after 300 seconds is $144.6 \mathrm{~ms}^{-1}$. Figure 2 shows the altitude of the aircraft and module. Apart from the obvious oscillations at times when the aircraft pitches up and down and the payout of the cable increases or decreases, the module follows the aircraft flight closely. Note that initially the aircraft and the module are separated by a cable length of 720 metres, but at the end of the reeling in process they are separated by a cable of length 30 metres.

The tension graph is shown in Figure 3. As soon as the aircraft pitches down at 20 seconds, the tension increases and the rate at which this tension grows is increased when the reeling in begins at $t=26$ seconds. At 30 seconds when the aircraft levels off, the tension decreases as the aircraft adjusts itself to the correct altitude. There is a decrease in tension at the aircraft end as the cable shortens. At 60 seconds, the aircraft pitches upwards and an initial decrease in the tension is seen, followed by a sharp increase. When the retrieval rate of the cable reduces to zero, the tension decreases and the oscillations become more apparent but rapidly decay. At 100 seconds, the tension increases and oscillates as the aircraft levels off to its straight path and the module oscillates about its equilibrium position. This oscillation diminishes as the module settles to its equilibrium position.

The second example is of a Boeing aircraft towing the module on a cable that is initially 5 metres in length and modelled with 10 segments. At time 10 seconds, the aircraft banks at $20^{\circ}$ at $4^{\circ}$ per second. Payout of the cable is commenced at time $t=30$ seconds, and reaches the maximum rate of $10 \mathrm{~ms}^{-1}$ at $t=35$ seconds where after this rate is maintained. Then at 150 seconds, the aircraft banks to a reduced angle of $10^{\circ}$ at a rate of $-4^{\circ}$ per second. At the same time, the aircraft pitches up to $10^{\circ}$ at $2^{\circ}$ per second with full power. The aircraft is then returned to level flight path 30 seconds later with the 


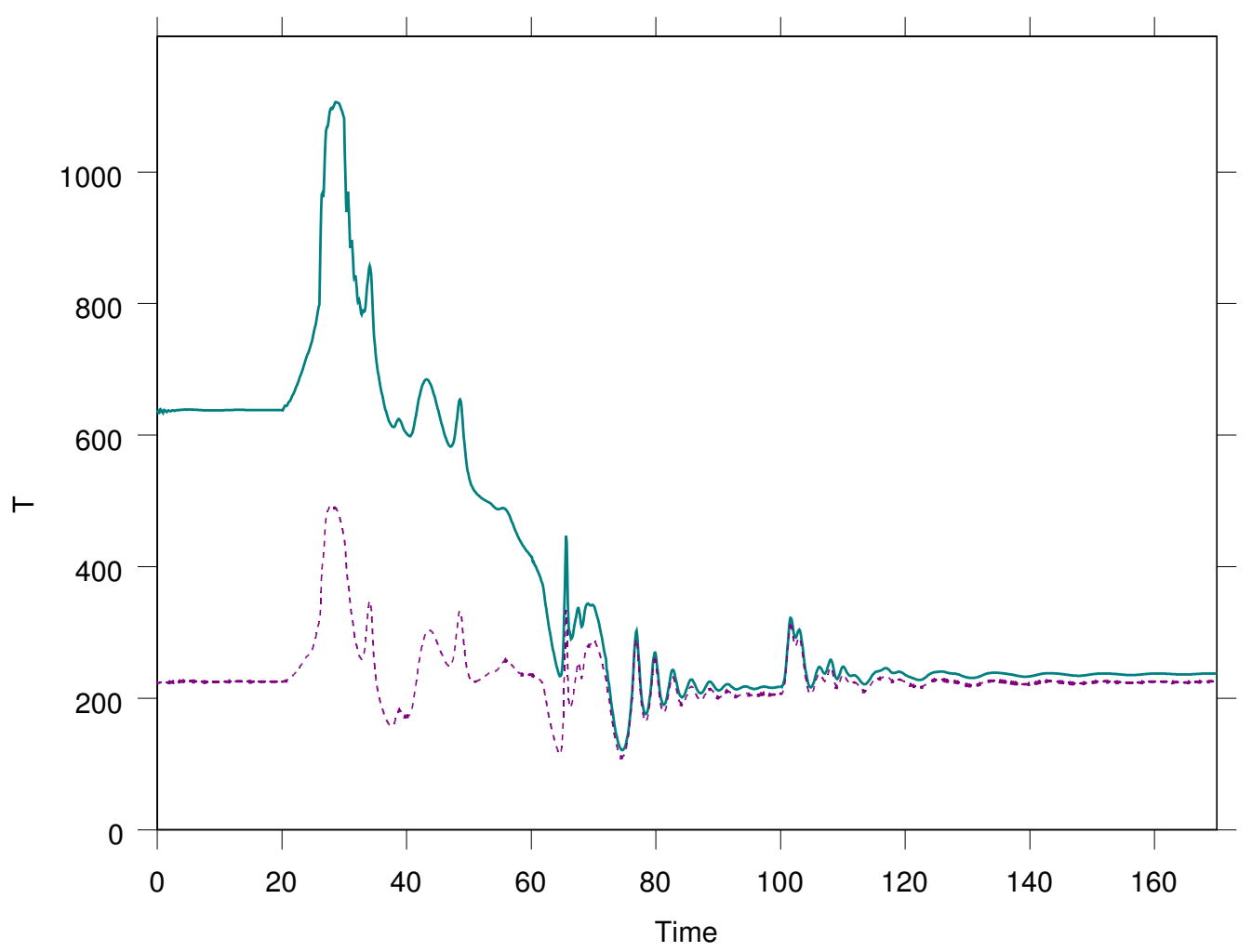

Figure 3: Tension of the cable at the aircraft end (green solid line) and module end (purple dashed line). 


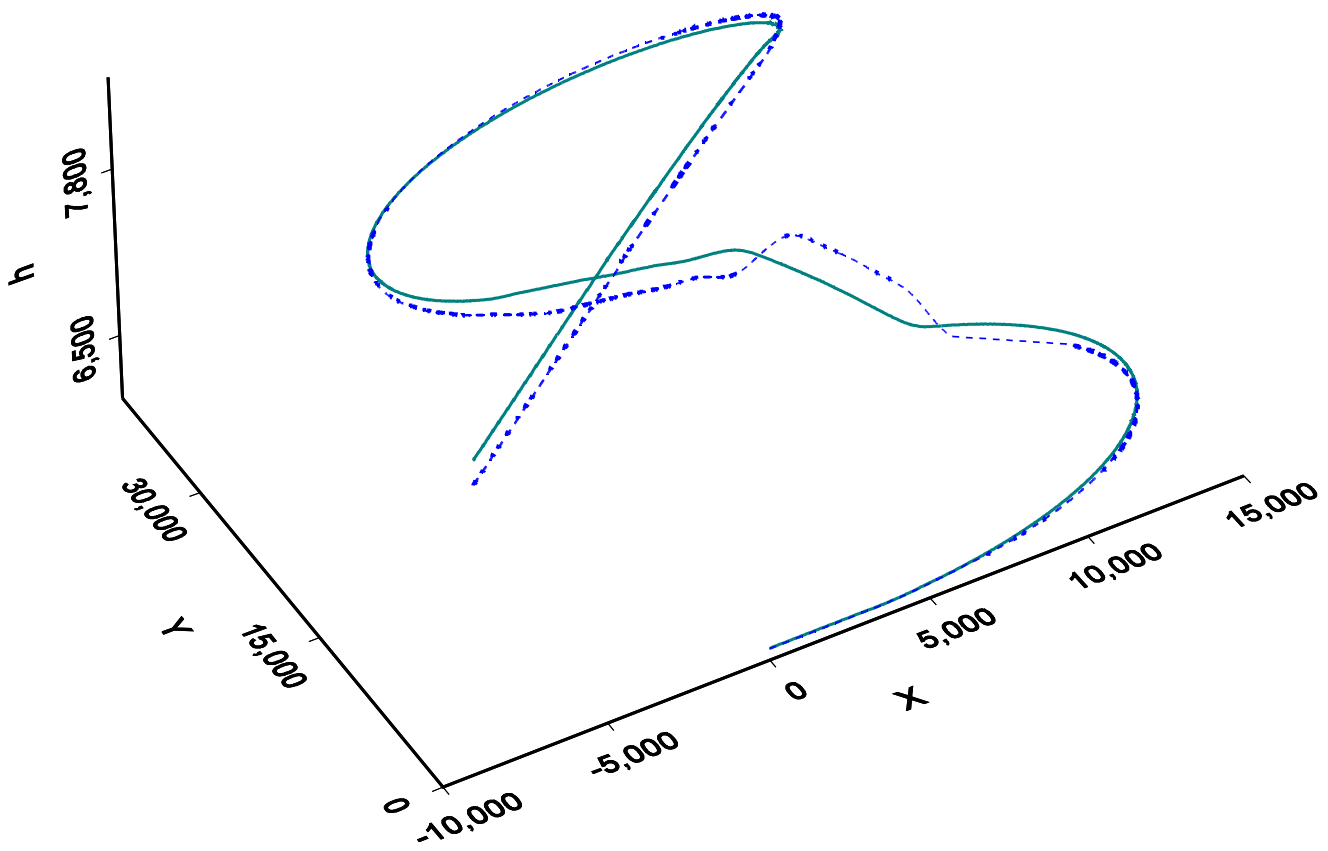

Figure 4: Position of the Boeing aircraft (green solid line) and the towed module (blue dashed line) during the payout process.

throttle back to its initial position. At 220 seconds, the aircraft banks in the opposite direction at an angle of $40^{\circ}$ at $5^{\circ}$ per second and pitches at $5^{\circ}$ with a rate of $1^{\circ}$ per second with full power. At 230 seconds, the cable's payout rate is reduced to zero in the next 5 seconds. Finally, at 350 seconds, the aircraft levels off and continues to fly in a straight and horizontal path. A total of 2000 metres of cable is payed out in the process. At the end of the simulation, it has a stretched length of 2011 metres (which includes the initial 5 metres of cable) and is modelled by 51 segments. At the end of the flight, the aircraft speed has increased to $233.9 \mathrm{~ms}^{-1}$.

Figure 4 shows a three-dimensional plot of this manoeuvre and the alti- 
tude of the aircraft and module is shown in Figure 5. Figure 6 shows the tension decreases initially as the aircraft starts to turn. Once the payout velocity has reached the steady rate of $10 \mathrm{~ms}^{-1}$, the tension increases linearly. At 150 seconds, the aircraft banks at a reduced angle and pitches up. This causes a vertical oscillation of the module which induces an oscillation in the tension. The tension again oscillates when the aircraft banks in the opposite direction and increases as the aircraft pitches down to a lower angle. When the payout rate of the cable is reduced at 230 seconds, the tension again increases, and once the payout ceases 5 seconds later, the tension decreases slightly but then increases again as the aircraft continues to turn and climb. When the aircraft levels off at about 350 seconds, the tension decreases slightly then tends to a constant value.

\section{Conclusions}

A simple method of integrating two models, one a relatively simple model of an aircraft that employs a variable time step, and the other a complex model of a cable with an attached body which uses a larger fixed time step, has been described and shown to perform well. If accuracy was the only consideration, clearly the best way to integrate the two models would be to add the discretized equations that govern the motion of the aircraft to the system of equations solved at each time step, but then the large system of non-linear equations would need to be solved much more often as a small time step would be needed. The computational time would then be prohibitive.

\section{References}

[1] Bourmistrov, A., Feedback Control Design For Aerial Towed Systems, RMIT Aerospace Engineering Department PhD. thesis, 1997. C624 


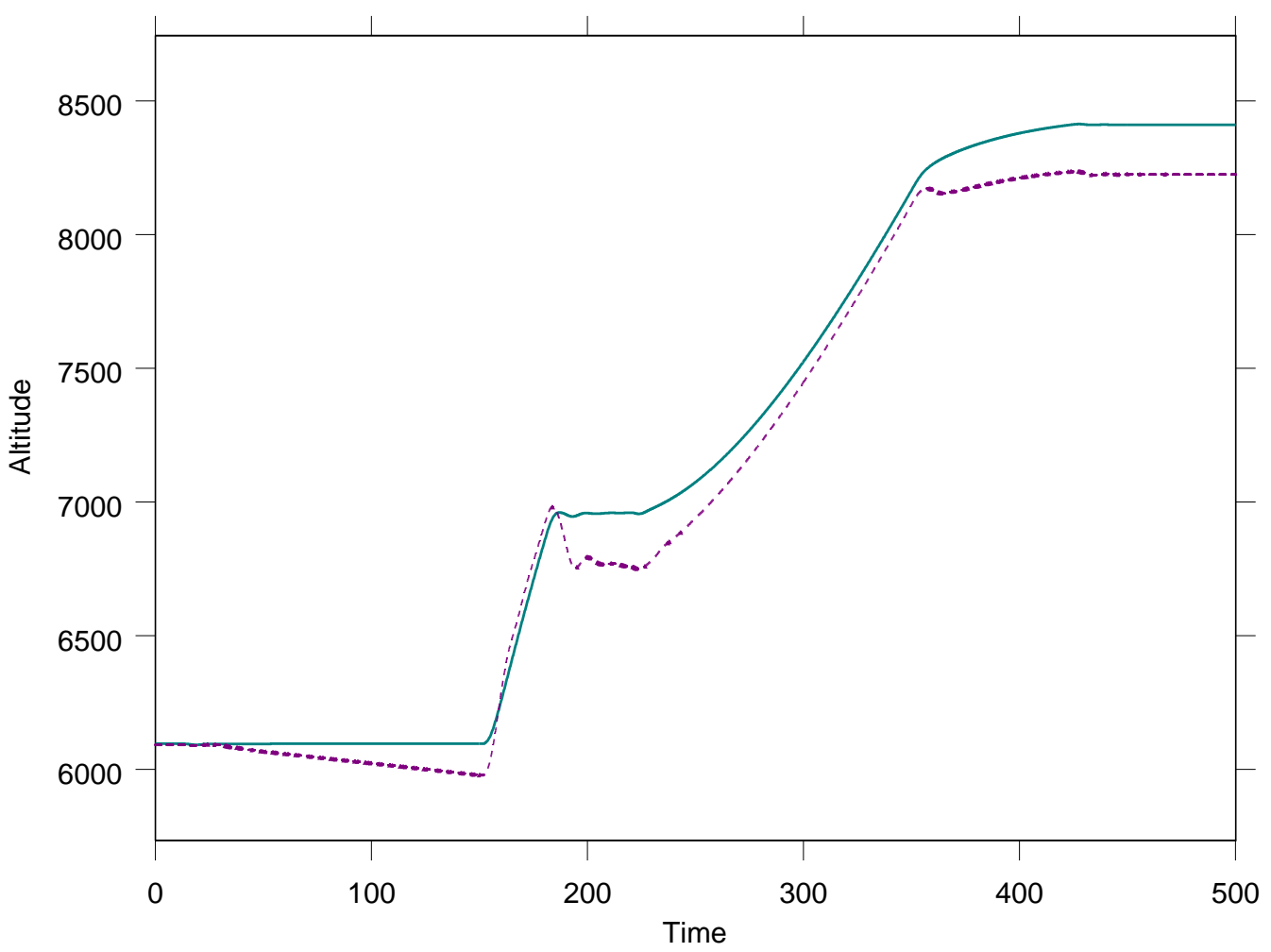

FIgURE 5: Variation of height over time for the Boeing aircraft (green solid line) and the towed module (purple dashed line). 


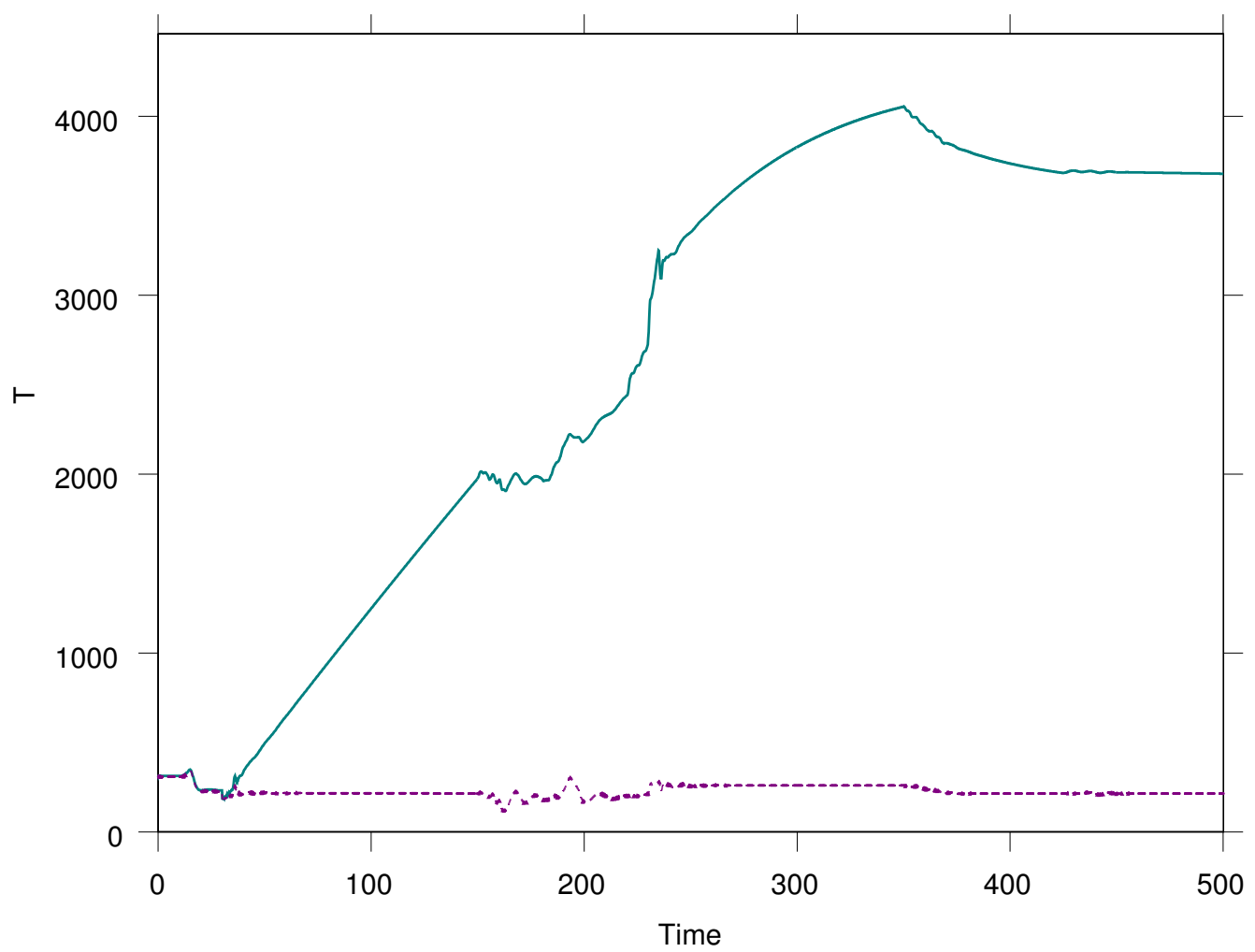

Figure 6: Cable tension at the aircraft end (green solid line) and the towed module end (purple dashed line). 
[2] Burden, R. L. and Faires, J. D., Numerical Analysis, Seventh edition, Brooks-Cole, 2001. C616, C617

[3] Chin, C. K. H., May, R. L. and Connell, H. J., Numercial Simulation of the Manoeuvring of an Aircraft, Proceedings of the Fourth Biennial Engineering Mathematics and Applications Conference, editors May, R. L., Fitz-Gerald, G. F. \& Grundy, I. H., The Institution of Engineers, Australia, pp. 91-94, 2000. C617

[4] Chin, C. K. H., May, R. L. and Connell, H. J., A Numercial Model of a Towed Cable-Body System, The Anziam Journal, Vol. 42 Electronic Supplement, pp. C362-C384, 2000. http://anziamj . austms.org.au/V42/CTAC99/Chin C616, C617

[5] Cochran, J. E. Jr., Innocenti, M., No, T. S. \& Thukral, A., Dynamics and Control of Maneuverable Towed Flight Vehicles, Journal of Guidance, Control and Dynamics, Vol. 15, No. 5, pp. 1245-1252, 1992. C623, C624

[6] Roskam, J., Airplane flight dynamics and automatic flight controls, Roskam Aviation and Engineering Corp, 1982. C616, C621, C624

[7] Wingham, P. J. and Ireland, B., The dynamic behaviour of towed airborne and underwater body-cable systems, Advances in Underwater Technology, Ocean Science and Offshore Engineering, Vol. 15, 1988. C623, C624 